\title{
Intensive Phase
}

National Cancer Institute

\section{Source}

National Cancer Institute. Intensive Phase. NCI Thesaurus. Code C85515.

The first 8 weeks of treatment for tuberculosis during which the infection is treated aggressively with multiple drugs. 